\title{
A Software Tool for Visualizing, Managing and Eliciting SWRL Rules
}

\author{
Saeed Hassanpour, Martin J. O’Connor, and Amar K. Das \\ Stanford Center for Biomedical Informatics Research \\ MSOB X215, 251 Campus Drive, Stanford, California, USA 94305 \\ \{saeedhp, martin.oconnor, amar.das\} astanford.edu
}

\begin{abstract}
SWRL rule are increasingly being used to represent knowledge on the Semantic Web. As these SWRL rule bases grows larger, managing the resulting complexity can become a challenge. Developers and end-users need rule management tools to tackle this complexity. We developed a rule management tool called Axiomé that aims to address this challenge. Axiomé support the paraphrasing of SWRL into simple English, the visualization of the structure both of individual rules and of rule bases, and supports the categorization of rules based on an analysis of their syntactic structure. It also supports the automatic generation of rule acquisition templates to facilitate rule elicitation. Axiomé is available as a plugin to the Protégé-OWL ontology development environment.
\end{abstract}

Keywords: Rule Management, Rule Elicitation, Rule Visualization, Rule Paraphrasing, Rule Categorization, OWL, SWRL.

\section{Introduction}

SWRL rules [4] are increasingly being used to represent knowledge on the Semantic Web. As the size of rule bases increases, users can face problems in understanding the content of these rule bases and managing the complexity of resulting knowledge. To support this rule management task, and to assist in the rapid exploration and understanding of rule bases, we have developed a tool that assists in the interpretations of rule bases through the generation of high-level abstractions of rule base structure. In particular, this software uses rule paraphrasing and rule visualization to help nonspecialists understand and manage potentially complex rule bases. It also supports the automatic detection of common patterns in rule bases through the categorization of rules into related groups. These categorizations are used as a basis of rule elicitation functionality that assists non-specialists in developing new rules.

This tool is called Axiomé [3] and was developed as a plug-in to the popular Protégé-OWL ontology development environment. It provides an array of tools for managing SWRL rule bases in OWL ontologies. It supports visual rule base exploration, automated rule categorization, rule paraphrasing, and rule elicitation functionality with the goal of facilitating the management of large SWRL rule bases. 


\section{Axiomé: Core Features}

Axiomé has five main functional areas, which are available as sub-tabs within the plug-in: (1) a Rule Graph tab that provides a graph structure to browse and explore the SWRL rule base; (2) a Rule Visualization tab to visualize individual rules; (3) a Rule Paraphrasing tab that displays an English-like text explanation for each rule; (4) a Rule Categorization tab to automatically categorize rules in a rule base; and (5) a Rule Elicitation tab that provides a graphical templates to acquire new rules based on analysis of existing rules in a rule base. A Rule Browser component is permanently displayed to show a tree-table representation of the SWRL rules in an ontology. This tree-table enables users to explore the rule base and launch any of five sub-tabs for the rule or group being explored.

\subsection{Rule Graph}

The Rule Graph tab provides a graphical representation of a SWRL rule base (Figure 1). Each rule in the rule base is shown as a node; edges between two nodes indicate the SWRL atoms shared by the rules and the dependency direction. A number of graphical layouts are supported. The JUNG visualization framework [2] was used to generate graph layouts. Search functionality is also provided. Rules matching a search term can be visually highlighted in the graph. Rule groups and dependencies between rules can also be indicated visually. Different types of dependency groups can be filtered and indicated separately in the dependency graph. Also, cyclical dependencies between rules can be found and visually highlighted.

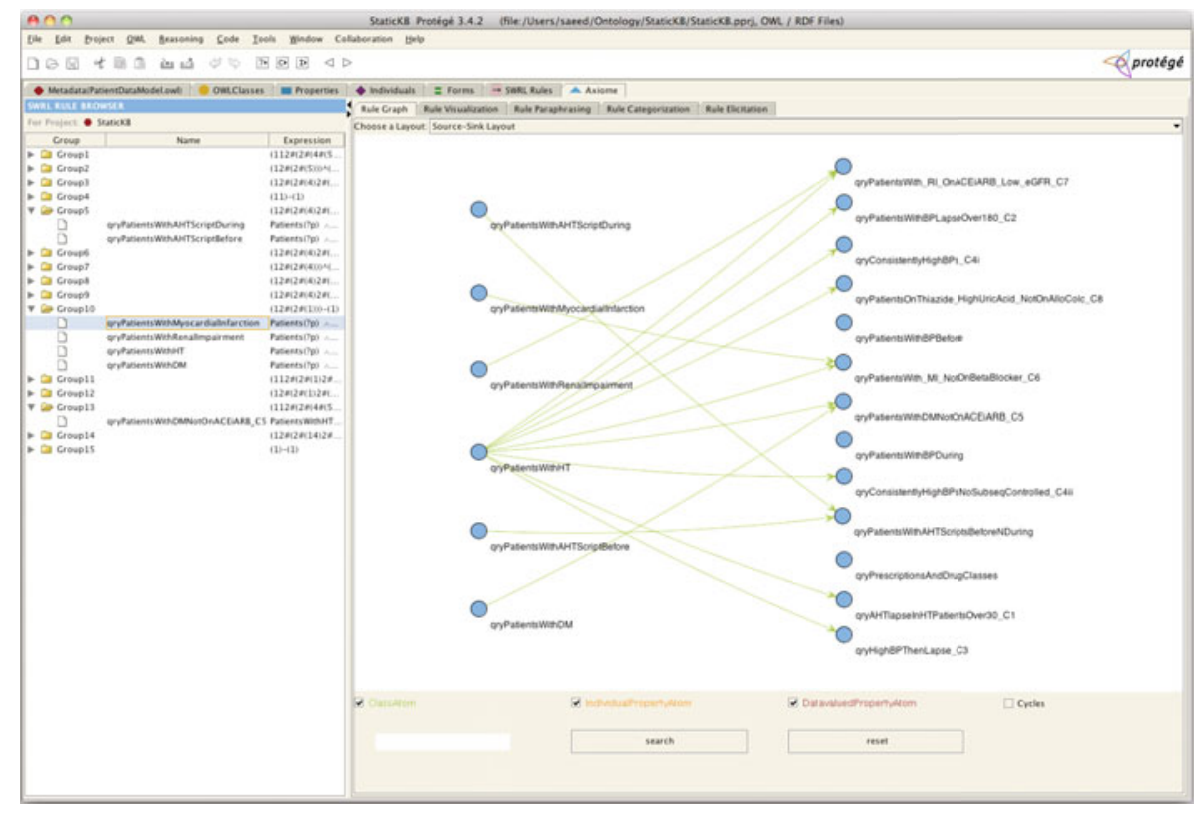

Fig. 1. An example of a rule base visualization in Axiome's Rule Graph tab. This visualization shows the dependencies between rules in a clinical care auditing rule base. 


\subsection{Rule Visualization}

The Rule Visualization tab (Figure 2) allows the arrangement of atoms in a rule to be visualized as a tree structure. These trees are generated by performing a depth-first search for each variable chain in the rule. A number of heuristics are employed to ensure that the most significant clauses in a rule are given more prominence [1].

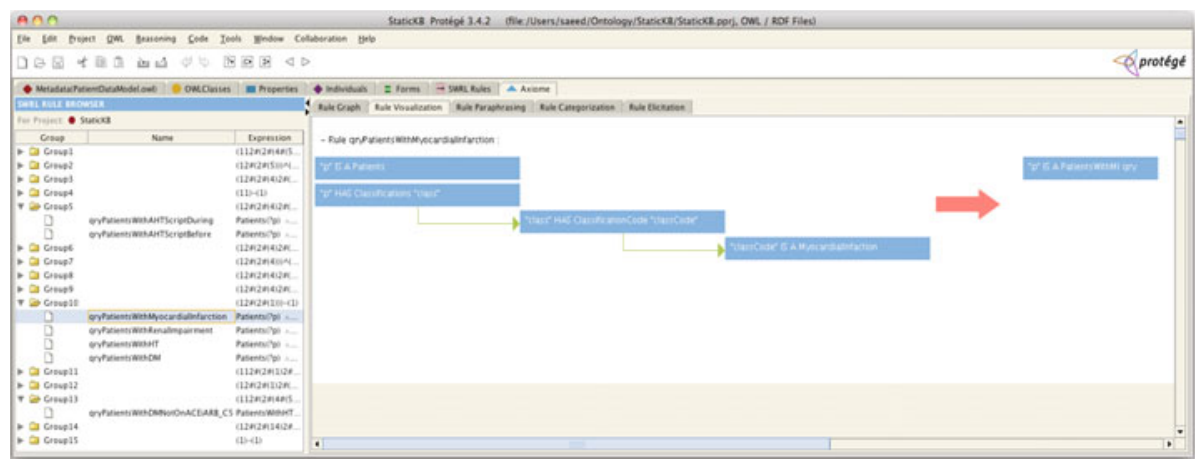

Fig. 2. An example of the visualization of a simple rule in the Rule Visualization tab

\subsection{Rule Paraphrasing}

The Rule Paraphrasing tab (Figure 3) uses a similar approach to build a tree structure for each rule and then uses additional heuristics to generate understandable English paraphrases of that rule [1].

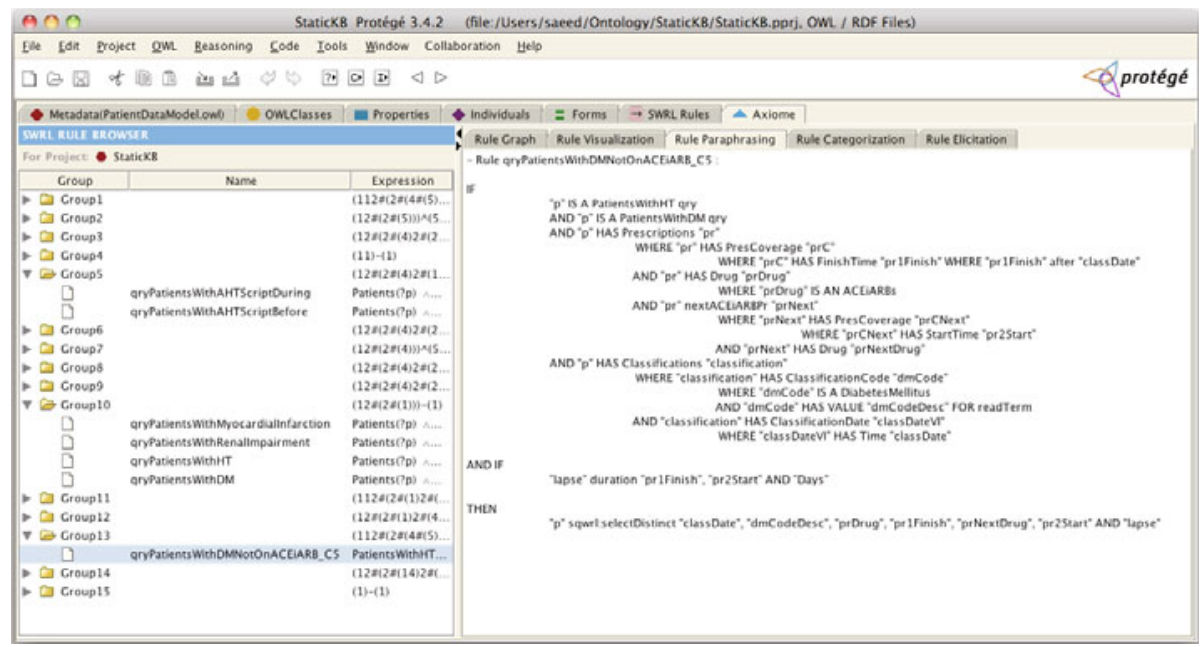

Fig. 3. An example of rule paraphrase generated by Axiome's Rue Paraphrasing tab. The rule is selected from a clinical care auditing rule base. 


\subsection{Rule Categorization}

The Rule Categorization tab uses the data structure that is generated for the rule visualization and paraphrasing tabs to automatically group rules with a similar syntactic structure [1]. It then graphically displays the results of this grouping. These groupings can then be used in the Rule Graph tab when exploring the rule base.

\subsection{Rule Elicitation}

The Rule Elicitation tab (Figure 4) provides graphical rule templates to facilitate acquisition of rules. It generates these templates using the syntactic structures generated for rule grouping. Users can select an appropriate rule group and then generate a graphical acquisition template to enter rules with the structure of other rules in that group.

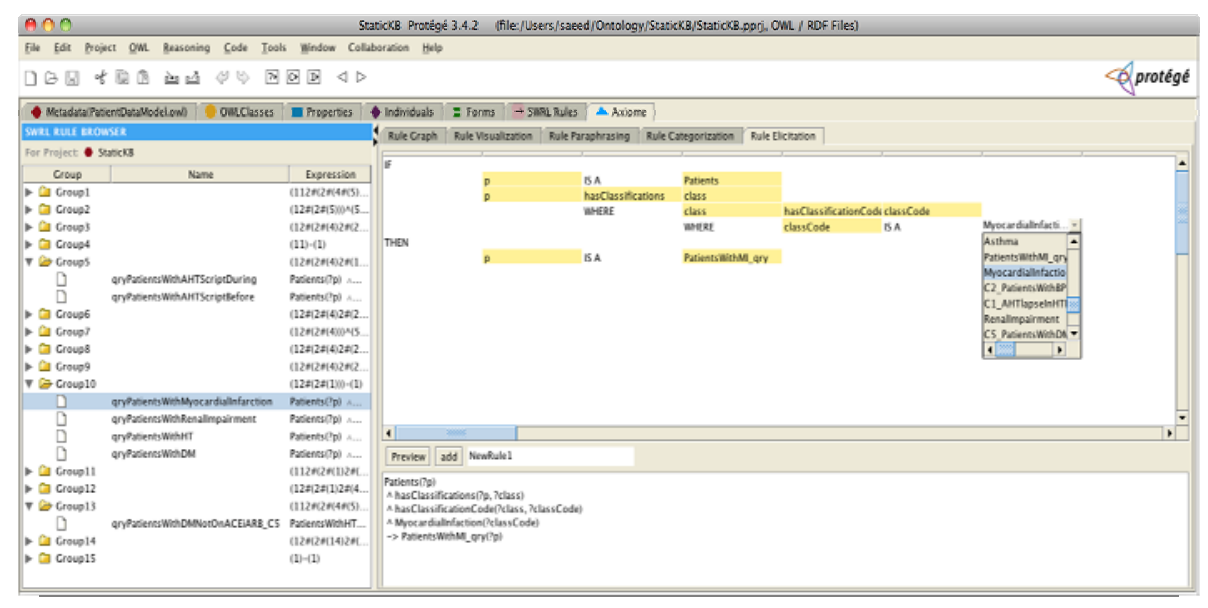

Fig. 4. Screen shot of Axiome's Rule Elicitation tab. This tab can automatically generate a rule acquisition template from an existing rule group.

\section{Summary}

We have described Axiomé, a free, open-source Protégé-OWL plug-in to support SWRL rule management. We have shown how the various features of the tool can facilitate understanding of SWRL rule bases through rule base graphing, rule visualization, and rule paraphrasing. The tool also supports the extension of rule bases through the elicitation of new rules using rule templates. It is included as one the default plug-ins in the latest Protégé-OWL 3.4 release [3]. We are currently testing its utility in the development and management of biomedical rule bases. We are also implementing a web-based version, which will to be available as a plugin to Web Protégé. 


\section{References}

1. Hassanpour, S., O’Connor, M.J., Das, A.K.: Exploration of SWRL Rule Bases through Visualization, Paraphrasing, and Categorization of Rules. In: Governatori, G., Hall, J., Paschke, A. (eds.) RuleML 2009. LNCS, vol. 5858, pp. 246-261. Springer, Heidelberg (2009)

2. Java Universal Network/Graph Framework, http://jung. sourceforge. net

3. Axiomé, http://protegewiki.stanford.edu/index.php/Axiomé

4. SWRL Submission, http://www.w3 .org/Submission/SWRL

5. Clinical-care auditing ontology, http://www.cs.auckland.ac.nz/ thusitha/aiim09/ 